\title{
Dronedarone does not affect infarct volume as assessed by magnetic resonance imaging in a porcine model of myocardial infarction
}

\author{
JOSEFINE LINKE ${ }^{1 *}$, KIRSTEN UTPATEL $^{2 *}$, CARMEN WOLKE $^{1}$, MATTHIAS EVERT $^{2}$, JENS-PETER KÜHN $^{3}$, \\ ALICJA BUKOWSKA ${ }^{4}$, ANDREAS GOETTE ${ }^{4,5}$, UWE LENDECKEL ${ }^{1}$ and BARBARA PETERS ${ }^{6}$ \\ ${ }^{1}$ Institute of Medical Biochemistry and Molecular Biology; ${ }^{2}$ Institute of Pathology; ${ }^{3}$ Department of \\ Diagnostic Radiology and Neuroradiology, University Medicine Greifswald, Ernst-Moritz-Arndt-University, \\ Greifswald D-17487; ${ }^{4}$ EUTRAF Working Group, Molecular Electrophysiology, University Hospital Magdeburg, \\ Magdeburg D-39120; ${ }^{5}$ Department of Cardiology and Intensive Care Medicine, St. Vincenz-Hospital, \\ Paderborn D-33098; ${ }^{6}$ Institute of Physiology, University Medicine Greifswald, \\ Ernst-Moritz-Arndt-University, Karlsburg D-17495, Germany
}

Received September 29, 2014; Accepted May 28, 2015

DOI: $10.3892 / \mathrm{mmr} .2015 .4077$

\begin{abstract}
Dronedarone has been demonstrated to be harmful in patients with recent decompensated heart failure. Furthermore, a PALLAS study reported that dronedarone therapy increases mortality rates in patients with permanent atrial fibrillation. Although a pathophysiological explanation for these finding remains to be elucidated, the long term effects of dronedarone on myocardial structure and stability have been suggested. The aim of the present study was to determine whether dronedarone therapy affects left ventricular (LV) function in a chronic model of myocardial infarction (MI). An anterior MI was induced in 16 pigs. Of these animals, eight pigs were then treated with dronedarone for 1 week prior to, and 4 weeks following MI, the remaining pigs served as controls. LV angiography was performed 4 weeks after MI to determine the LV ejection fraction (LVEF). A post-mortem magnetic resonance imaging scan of the LV was then performed on the two groups $(n=6)$ to determine the volume and size of the induced MI. Dronedarone therapy did not affect systemic and intracardiac hemodynamic parameters or LVEF during the follow-up assessment. Of note, dronedarone had no negative effect on the total infarct volume and size and did not induce lethal proarrhythmic effects following the induced anterior MI. Therefore,
\end{abstract}

Correspondence to: Professor Uwe Lendeckel, Institute of Medical Biochemistry and Molecular Biology, University Medicine Greifswald, Ernst-Moritz-Arndt-University, Ferdinand-Sauerbruch-Strasse, Greifswald D-17475, Germany

E-mail: uwe.lendeckel@uni-greifswald.de

*Contributed equally

Key words: dronedarone, infarct volume, magnetic resonance imaging, myocardial infarction, porcine model the results suggested that dronedarone did not increase the volume or size of induced anterior MI and did not affect LV performance. Thus, dronedarone therapy was observed to be safe in a porcine model of anterior MI.

\section{Introduction}

Atrial fibrillation (AF) is associated with a high risk for subsequent ischemic events (1). Data from the previous ATHENA study demonstrated that dronedarone therapy reduces cardiovascular mortality rates, hospitalization and the incidence of myocardial infarction (MI) in patients with AF (2). Separate subgroup analyses revealed that the incidence of acute coronary syndromes is also reduced in patients with coronary artery disease at baseline (3). The pathological mechanisms underlying these observations remain to be elucidated.

In patients with $\mathrm{AF}$, the coronary flow reserve (CFR) is reduced (4,5). A compromised CFR has also been described in an acute model of rapid atrial pacing (RAP) in vivo, in which the fractional flow reserve appears to be unaffected $(6,7)$. Calcium overload and oxidative stress are likely to contribute to these effects $(6,7)$, as the two are associated with increased inflammation, fibrosis, adipocyte infiltration and myocardial hypertrophy and, thus, promote structural remodeling of the myocardium (8-10). In line with previous data demonstrating the potential of benzofuranes, including dronedarone (or amiodarone) to increase coronary flow $(11,12)$, our previous study demonstrated that dronedarone largely prevents the AF-dependent decline of the CRF during acute RAP in vivo (7). The capability of dronedarone to inhibit $\alpha$-adrenergic vasoconstriction $(13,14)$ may well contribute to the improved flow. In addition, as dronedarone reduces heart rate, an increase in diastolic duration and a reduction in oxygen demand may also contribute to its pleiotropic effects $(14,15)$. Of note, dronedarone has demonstrated cardioprotective activity in a porcine model of myocardial infarction/reperfusion, which is independent of the remaining subendocardial 
blood flow during ischemia (16). Although dronedarone has been observed to attenuate redox-activated and ischemia-associated gene expression (7), the mechanisms underlying its potential cardioprotective action remain to be fully elucidated. To confirm the hypothesis that the administration of dronedarone prevents functional changes in the coronary arteries and damage to the adjacent myocardial tissue, in vivo experiments are required, which allow for a pharmacological intervention. Consequently, the present study analyzed the possible effects of dronedarone on infarct size in a porcine model of acute MI with a subsequent 4 -week recovery period. This aimed to detect late structural/contractile alterations, which may result from the possible interference of dronedarone with structural remodeling and repair processes in the myocardium and infarct scar. These are known to be slow processes due to the long half-life time of the components involved, including matricellular proteins (17).

In this context, magnetic resonance imaging (MRI) may be a suitable approach to evaluate infarct size, as histological quantification is time consuming and its accuracy depends on the slice thickness. The 7 Tesla MRI method enables the potential to acquire three dimensional (3D) data with a thin slice thickness $(100 \mu \mathrm{m})$ and to quantify the 3D infarct structures at an almost microscopic level (18). Usually, contrast agents are used to identify necrotic tissue (19). However, these contrast agents may interfere with further biochemical or molecular biological analysis. To evaluate the capability of 7 Tesla MRI to differentiate between infarcted and non-infarcted myocardium at microscopic levels without the use of contrast agents, the present study performed 3D-MRI analysis and compared the results with microscopic findings of the corresponding regions.

\section{Materials and methods}

Anterior MI model. All animal care and experimental procedures were approved by the Landesverwaltungsamt Sachsen-Anhalt (Az42502-2-1117-IMTR). A total of 16 male castrated pigs (German Landrace/German Pietrain, mean weight $30 \mathrm{~kg}$, mean age 3 months; Schweinezucht Gladau $\mathrm{GmbH}$, Kleindemsin, Germany) were used in the current study. Animal health status was monitored daily prior to study and twice/day during the study. Animals were maintained between 15 and $20^{\circ} \mathrm{C}$ with a $12 \mathrm{~h} \mathrm{light/dark} \mathrm{cycle,} \mathrm{were} \mathrm{fed}$ with conventional piglet raising food (Deuka Deutsche Kraftfutterwerke GmbH \& Co., Düsseldorf, Germany) and had ad libitum access to water. The pigs were assigned into two study groups, both received the surgery-associated standard medication, described below, and one received dronedarone (Sanofi-Aventis, Paris, France). The dronedarone treatment (200 $\mathrm{mg}$ ) was administered orally twice a day, to attempt to match the quantity of $400 \mathrm{mg}$ used in the ATHENA trial (2), commenced 7 days prior to the experimental MI and was continued for 4 weeks until explantation of the heart. The experiments were performed in three temporally separated rounds, each containing six or four pigs each.

The premedication provided to the animals consisted of an oral administration of $100 \mathrm{mg}$ acetylsalicylic acid (Ratiopharm, Ulm, Germany) and 75 mg clopidogrel (Plavix ${ }^{\circledR}$, Sanofi Pharma, Berlin, Germany) 2 days prior to surgery, as well as a single dose of $25 \mathrm{mg}$ atenolol (Ratiopharm) 1 day prior to surgery. Atenolol is known to prevent mortality caused by severe arrhythmia during the induction phase of acute MI. For sedation, intramuscular injections of $0.2 \mathrm{ml}$ $\mathrm{kg}^{-1}$ ketamine (10\% ketamine; Ursotamin ${ }^{\circledR}$; Serumwerk Bernburg AG, Bernburg, Germany) and $0.1 \mathrm{ml} \mathrm{kg}^{-1}$ xylazine (2\% xylazine hydrochloride; Xylazin ${ }^{\circledR}$; Riemser Arzneimittel $\mathrm{GmbH}$, Greifswald, Germany) were administered. Anesthesia was induced using $6 \mathrm{ml}$ propofol intravenously (1\% Recofol, Curamed Pharma GmbH, Baden-Wurttemberg, Germany) and maintained with 1-2 vol\% isoflurane (Isofluran Curamed, Curamed Pharma GmbH) in a mixture of $\mathrm{O}_{2}: \mathrm{N}_{2} \mathrm{O}(30: 70 \mathrm{vol} \%)$ via an endotracheal tube. Oxygen saturation and electrocardiogram (ECG) were monitored continuously throughout the procedure. At the beginning of surgery, the animals received $0.04 \mathrm{ml} \mathrm{kg}^{-1}$ meloxicam subcutaneously $\left(20 \mathrm{mg} / \mathrm{ml}^{-1}\right.$ Metacam ${ }^{\circledR}$, Boehringer-Ingelheim GmBH, Ingelheim, Germany), $0.2 \mathrm{ml} / \mathrm{kg}^{-1}$ oxytetracycline subcutaneously $\left(10 \%\right.$ Ursocyclin ${ }^{\circledR}$, Serumwerk Bernburg AG), $250 \mathrm{mg}$ acetylsalicylic acid and 5,000 IU heparin intra-arterially (B. Braun Melsungen AG, Melsungen, Germany). Anticoagulatory prevention was prolonged with intra-arterial injections of 2,500 IU heparin during the procedure.

The MI was induced by temporary occlusion of the left anterior descending (LAD) artery using a balloon catheter (SeQuent ${ }^{\circledR}$ II; 3.0x15 mm; B. Braun Melsungen AG). Prior to occlusion, ventricular angiography and coronary angiography of the left main coronary artery were performed to examine the positions of its branches. To attain comparable infarct sizes, the occlusion was performed distal to the first septal branch. The correct position of the balloon catheter was confirmed using angiography (Artis Zee Biplane System; Siemens AG Healthcare Sector, Erlangen, Germany) (Fig. 1A). Prior to inflation of the balloon, $100 \mathrm{mg}$ lidocaine (Bela-pharm Arzneimittelfabrik, Vechta, Germany) and $0.5 \mathrm{mg}$ buprenorphine ( Temgesic ${ }^{\circledR}$, Essex Pharma GmBH, Munich, Germany) were administered intravenously. The blood flow of the LAD artery was interrupted for a total of $90 \mathrm{~min}$. Subsequently, the balloon catheter was deflated and the reperfusion phase was monitored for the first $60 \mathrm{~min}$.

After 4 weeks, the hearts were explanted for determination of the infarct size using MRI imaging (7.1 Tesla Animal MR Scanner; Bruker; Ettlingen, Germany). For surgery, the pigs were sedated, anesthetized and subjected to analgesia as described above. In addition, $1 \mathrm{mg}$ pancuronium (Ratiopharm) was administered intravenously for muscle relaxation. Following ventricular angiography, the pigs were sacrificed with $10 \mathrm{ml} \mathrm{KCl}$ intravenous administration. Following explantation, the hearts were fixed in $4.5 \%$ formalin for MRI imaging.

ECG monitoring and analysis. Throughout the entire infarction and explantation surgical procedures, the cardiac function of the pigs was monitored using a 6-lead-ECG (Axiom Sensis XP Monitoring System; Siemens AG Healthcare Sector) and by measurement of the intraaortic bloodpressure. In the resting ECG analysis, heart rate, PQ-duration (beginning of the P-wave to the beginning of the QRS-complex), QRS-duration and the QT-interval (beginning of the QRS-complex to the end of the T-wave) were investigated using an ECG lead II. The QT-duration was adjusted to the heart rate using Fridericia's 
A
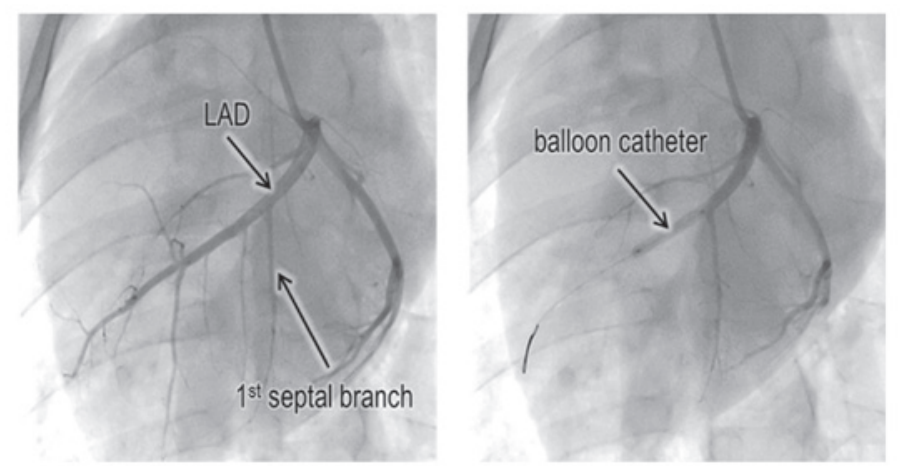

B

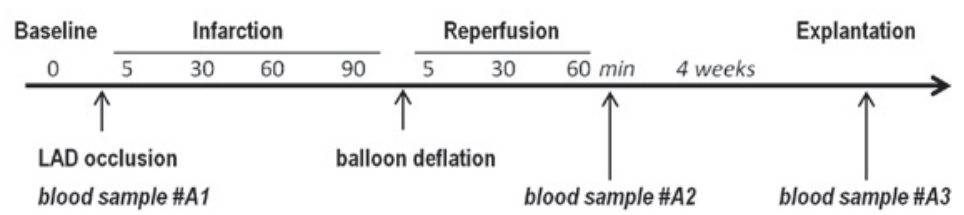

C

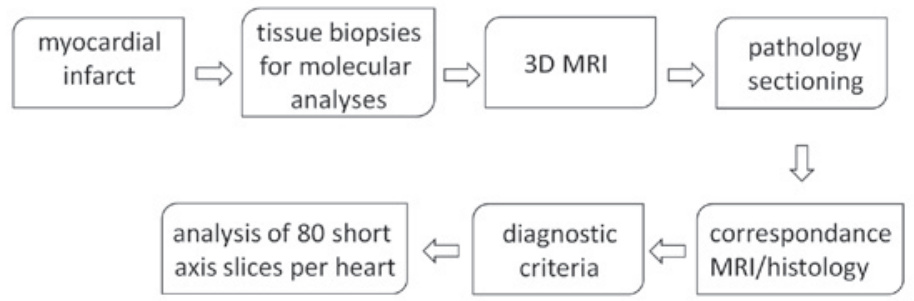

Figure 1. (A) Induction of anterior myocardial infarction by occlusion of the LAD artery using a balloon catheter. Coronary angiography was performed to inspect the branches of the LAD (left). The balloon catheter was positioned distal to the first septal branch and inflated (right). (B) Regimen of blood sample collection. (C) Work flow. LAD, left anterior descending.

formula $(\mathrm{QTc}=\mathrm{QT} / 3 \sqrt{\mathrm{RR}})$, as QTc, which appears to provide more accurate results, particularly at higher heart rates (20).

Blood and tissue collection. Blood samples were collected from the pigs prior to occlusion of the $\mathrm{LAD}, 60 \mathrm{~min}$ following reperfusion, and 28 days following infarction, as shown in Fig. 1B. Blood was collected into a BD Vacutainer ${ }^{\circledR}$ EDTA ( $6 \mathrm{ml}$; violet) or SST (5 ml; gold), respectively (BD Diagnostics, Heidelberg, Germany). The serum and plasma samples were prepared according to standard surgical procedures and were aliquoted, shock-frozen in liquid nitrogen and stored at $-80^{\circ} \mathrm{C}$ until further use. The plasma levels of troponin I (TnI), creatinine kinase (CK)-MB, alanine transaminase, aspartate aminotransferase (ASAT) and C-reactive protein were analyzed at the Institute of Clinical Chemistry and Laboratory Diagnostics of the University Medicine Greifswald (Greifswald, Germany) using an established platform and diagnostic kits, which are regularly applied in clinical routine analyses. Laboratory measurements were performed on a Siemens Vista 1500 analyzer (Siemens Healthcare Diagnostics, Eschborn, Germany). Aspartate (Siemens AST) and alanine aminotransferase (Siemens ALTI) activities were measured by adapted enzymatic methods of International Federation of Clinical Chemistry and Laboratory Medicine (Milan, Italy) recommended assays (21). The creatinine kinase isoenzyme MB activity was determined by an enzymatic method (Siemens CKMB) using an immunological inhibition of creatinine kinase M subunit activity (22). The cardiac troponin I concentration was measured by a homogenic, high-sensitivity sandwich chemiluminescence immunoassay based on the $\mathrm{LOCI}^{\mathrm{TM}}$ technology (Luminescent oxygen channeling immunoassay) (23).

Serum concentrations of dronedarone and its metabolite, SR35021, were measured following methanol/acetonitrile extraction by liquid chromatography-tandem mass spectrometry (LC-MS/MS), using a Shimadzu UFLC-XR (Shimadzu Corporation, Kyoto, Japan) coupled with an API4000 AB Sciex mass spectrometer).

For further biochemical and expression analyses, tissue samples of $\sim 0.5 \mathrm{~cm}^{3}$ were cut from the healthy left ventricular (LV) area and the right ventricular area, the border zone and the infarct area (MI). The precise localization of the samples to the indicated areas was confirmed using MRI and histological analyses. The tissue samples were then frozen in liquid nitrogen and stored at $-80^{\circ} \mathrm{C}$ for further use.

Determination of infarct size: Workflow, tissue processing and histopathological analysis. The general workflow of the investigation performed is outlined in Fig. 1C. Initially, the diagnostic criteria for MI required definition in the MRI images. In order to perform this verification, spatial correspondence between the MR images and the tissue-derived histology images is required. This is in general challenging due to the deformations involved during resection of the tissue and histological processing, and the difficulty of accurately registering the histological slices to the MR images (24). Therefore, following acquisition of the MRI 3D 
dataset, the hearts were cut into 5-7 slices, according to the areas of MI identified in the MRI short axis views. The slice thickness was $1.5 \mathrm{~cm}$. Biopsy sites were used to provide landmarks between MRI and pathology. Images were captured of each slice (Olympus E-410; Olympus, Tokyo, Japan) and the whole macroscopic infarct area, containing a rim of vital myocardium, was embedded in 2-6 paraffin blocks. Sections of $2 \mu \mathrm{m}$ were then cut vertically and mounted on glass slides (SuperFrost ${ }^{\circledR}, \mathrm{R}$. Langenbrinck, Emmendingen, Germany). The sections were subsequently deparaffinized with xylene and ethanol and stained with hematoxylin and eosin (HE) and acid fuchsin orange G (AFOG), according to standard protocols. The slices were examined using a Zeiss microscope (Axioplan2, Zeiss, Jena, Germany). To outline the infarcted myocardium, AFOG staining was selected, as it not only distinguishes between normal myocardial cells and areas of fibrosis, but also detects non-vital myocardial cells. In AFOG staining, fibrosis of the infarcted area stains blue. Residual red-stained non-vital cardiomyocytes were occasionally found within the fibrotic areas, but not in the surrounding tissue. The border between the infarcted and vital tissues were sharp and readily identified in each sample. The AFOG-stained specimens were then scanned (Leica SCN 400 slide scanner; Leica Microsystems GmbH, Wetzlar, Germany) and the images pasted into the images of the respective slice using Adobe Photoshop CS6 (Adobe Systems Europe, Ltd., Maidenhead, UK). A significant difficulty encountered was that of tissue deformation caused during histological processing. Therefore, the pasted histology slices were aligned to fit to the macroscopic image. In the resultant image, as well as in the corresponding MRI section, the infarct area was measured using ImageJ software (National Institutes of Health Bethesda, MA, USA). A good correlation between the relative area of the infarct (\% of LV area) in the MRI images and the corresponding histological images was observed $(\mathrm{R}=0.98 ; \mathrm{n}=40)$.

Hemodynanic data. The LV end-diastolic volume, stroke volume and ejection fraction were determined using LV angiography. The ventricular angiography was performed at a $30^{\circ}$ right anterior oblique projection using a $6 \mathrm{~F}$ pigtail catheter (B. Braun Melsungen AG), which was moved into the left ventricle through an arterial $7 \mathrm{~F}$ port (B. Braun Melsungen AG). The injection of the contrast medium, iopromid (Ultravist ${ }^{\circledR}-370$; Bayer Healthcare, Berlin, Germany) was performed with an angiomat (Angiomat 3000; Liebel-Flarsheim Company LLC, Cincinnati, OH, USA), using a volume of $20 \mathrm{ml}$ contrast medium and a flow rate of $12 \mathrm{ml} / \mathrm{sec}$.

Quantitative analysis of the LV volumes was performed using the image data processing software of the Artis Zee (Siemens Healthcare, Berlin, Germany) X-ray system for interventional radiology. For this purpose, all images were automatically calibrated with an isocenter calibration. Subsequently, the end-diastolic and end-systolic volume were determined by an automatic contour detection and subsequent manual correction, based on images captured of the left ventricle in end-diastole end-systole. Using these data, the Artis Zee software (Siemens Healthcare) calculated the LV end-diastolic and end-systolic volumes using the biplane area length method, the stroke volume as the difference of the end-diastolic volume, and the end-systolic volume and ejection fraction as a quotient of the stroke volume and the end-diastolic volume. All data were collected in an analysis report, which was stored digitally together with the angiography images.

Calculation of infarct volume and size using 3D MRI. Imaging of the formalin fixed hearts was performed on a 7.1 Tesla Animal MR Scanner. A three-dimensional gradient echo sequence was used; repitition time/echo time, $30 / 5 \mathrm{~ms}$; interpolated matrix, 1,024x1,024 mm; field of view, $110 \mathrm{~mm}$; 144 slices, slice thickness, $0.7-\mathrm{mm}$.

From the 3D MRI dataset, a stack of $1 \mathrm{~mm}$ thick short axis slices, rectangular to the ventricular septum and covering the whole ventricle, were generated. A total of 70-80 slices were evaluated, depending on the size of the heart, using Osirix software (Pixameo, Bernex, Switzerland) by two observers in a blinded-manner. All measurements were performed manually. LV volume and mass, determined as mass $(\mathrm{g})=$ volume $(\mathrm{ml}) \times$ myocardial density $(1.05 \mathrm{~g} / \mathrm{ml})$, was calculated from the difference between the LV pericardial volume and endocardial volume. The infarct size was expressed as the absolute volume and as the LV infarct index, denoting the infarct mass as a percentage of the LV mass. Biopsy had been performed on all hearts in the infarct and peri-infarct regions. The infarct region was estimated in these areas using different image planes and alike planes, tangential to the myocardial wall.

Thinning of the infarcted ventricle wall is not always accompanied by complete scarring of the region (25). For this reason, the area of $\mathrm{LV}$ infarct as a percentage of the $\mathrm{LV}$ area was measured in addition to the infarct volume associated with the LV volume.

Statistical analysis. The values in Table I are presented as the median with 1st and 3rd quartiles, normalized to the median of the experimental round. The Mann-Whitney-U test for unpaired group comparison was performed to determine any differences between the control and dronedarone at the baseline, during the procedure $(5,30,60$ and $90 \mathrm{~min}$ of infarction; 5, 30 and 60 min of reperfusion) and after 4 weeks (explantation). To determine time-dependent changes within one experimental groups, the Wilcoxon-test for paired group comparisons was performed. A Bland-Altman plot was used for analyzing the associations between infarct size determination by MRI and pathohistology. All statistical analyses was two-tailed. $\mathrm{P}<0.05$ was considered to indicate a statistically significant difference, without $\alpha$-adjustment. All data are expressed as the median with box (1st and 3rd quartile) and whisker (range) plots. Statistical analyses were performed using IBM ${ }^{\circledR}$ SPSS $^{\circledR} 20$ (IBM SPSS, Somers, NY, USA).

\section{Results}

Dronedarone serum levels. To prevent any failure of treatment due to too low blood levels of dronedarone, a high dosage (200 mg) was administered. Blood levels of dronedarone or its metabolite, SR35021, were $58.9 \pm 6.9$ or $8.1 \pm 0,73 \mathrm{ng} / \mathrm{ml}$, respectively, in the dronedarone-treated group and, thus confirmed to be in the therapeutic range $\left(\mathrm{C}_{\max }=111 \mathrm{ng} / \mathrm{ml}\right)$. As expected, 
Table I. Hemodynamic parameters during infarction and reperfusion.

\begin{tabular}{|c|c|c|c|c|}
\hline Parameter & Time & $\begin{array}{l}\text { Control median } \\
\text { (1st; 3rd quartile) }\end{array}$ & $\begin{array}{l}\text { Dronedarone median } \\
\text { (1st; 3rd quartile) }\end{array}$ & $\begin{array}{c}\text { P-value } \\
\text { (Mann-Whitney-U) }\end{array}$ \\
\hline \multirow[t]{2}{*}{ Body weight (kg) } & Baseline & $31.78(30.31 ; 33.18)$ & $30.65(29.54 ; 31.82)$ & 0.050 \\
\hline & Infarction & $38.73(37.38 ; 39.25)$ & $39.24(37.62 ; 40.14)$ & 0.505 \\
\hline \multirow{9}{*}{$\begin{array}{l}\text { Heart rate } \\
\text { (beats/min) }\end{array}$} & Baseline & $98.50(95.99 ; 104.09)$ & $97.50(91.76 ; 99.89)$ & 0.645 \\
\hline & $5 \mathrm{~min} \operatorname{Inf}$ & $91.67(83.92 ; 110.64)$ & $93.00(88.62 ; 99.73)$ & 0.574 \\
\hline & $30 \min \operatorname{Inf}$ & $98.11(90.86 ; 115.68)$ & $97.00(91.66 ; 102.34)$ & 1.000 \\
\hline & $60 \min \operatorname{Inf}$ & $93.48(83.98 ; 105.35)$ & $94.52(91.42 ; 102.78)$ & 0.779 \\
\hline & $90 \min \operatorname{Inf}$ & $89.93(82.18 ; 100.53)$ & 93.03 (88.90; 95.10) & 0.328 \\
\hline & 5 min Reperf & $123.83(115.36 ; 134.27)$ & $120.52(106.82 ; 126.77)$ & 0.536 \\
\hline & 30 min Reperf & $138.13(123.09 ; 143.59)$ & $143.68(131.41 ; 156.44)$ & 0.414 \\
\hline & 60 min Reperf & $119.13(108.30 ; 123.93)$ & $132.11(107.89 ; 154.13)$ & 0.336 \\
\hline & Explantation & $87.50(76.25 ; 109.25)$ & $95.72(89.05 ; 123.81)$ & 0.132 \\
\hline \multirow{9}{*}{$\begin{array}{l}\text { Mean arterial blood } \\
\text { pressure }(\mathrm{mmHg})\end{array}$} & Baseline & $101.07(95.96 ; 114.66)$ & $102.42(94.82 ; 108.13)$ & 0.959 \\
\hline & $5 \mathrm{~min} \operatorname{Inf}$ & $60.39(56.70 ; 83.33)$ & $65.61(56.67 ; 72.10)$ & 0.798 \\
\hline & $30 \mathrm{~min} \operatorname{Inf}$ & $70.66(63.66 ; 75.73)$ & $74.16(71.84 ; 76.74)$ & 0.336 \\
\hline & $60 \min \operatorname{Inf}$ & $70.50(62.32 ; 74.21)$ & $66.70(61.65 ; 70.70)$ & 0.345 \\
\hline & $90 \min \operatorname{Inf}$ & $64.50(57.50 ; 66.27)$ & $66.05(63.95 ; 71.82)$ & 0.397 \\
\hline & 5 min Reperf & $62.00(56.50 ; 78.34)$ & $59.00(48.27 ; 72.00)$ & 0.536 \\
\hline & 30 min Reperf & $65.00(59.50 ; 74.50)$ & $66.00(52.70 ; 71.44)$ & 0.573 \\
\hline & 60 min Reperf & $65.97(62.56 ; 82.60)$ & $63.06(60.15 ; 76.17)$ & 0.336 \\
\hline & Explantation & $98.50(58.00 ; 118.25)$ & $97.38(78.23 ; 122.44)$ & 0.818 \\
\hline \multirow[t]{2}{*}{ PQ-duration (ms) } & Baseline & $100(100 ; 113)$ & $100(96 ; 104)$ & 0.442 \\
\hline & Explantation & $100(100 ; 109)$ & $100(100 ; 110)$ & 0.878 \\
\hline \multirow[t]{2}{*}{ QRS-duration (ms) } & Baseline & $55.0(54.1 ; 57.9)$ & $57.0(54.1 ; 57.9)$ & 0.798 \\
\hline & Explantation & $60.0(57.0 ; 63.0)$ & $60.0(60.0 ; 64.0)$ & 0.574 \\
\hline \multirow[t]{2}{*}{ QTc-duration (ms) } & Baseline & $362(348 ; 381)$ & $376(353 ; 396)$ & 0.382 \\
\hline & Explantation & $399(391 ; 405)$ & $410(398 ; 430)$ & 0.161 \\
\hline \multirow{2}{*}{$\begin{array}{l}\text { LV end-diastolic } \\
\text { volume }(\mathrm{ml})\end{array}$} & Baseline & $80.64(77.13 ; 91.57)$ & $75.58(70.47 ; 79.57)$ & 0.105 \\
\hline & Explantation & $101.40(91.36 ; 109.94)$ & $108.88(96.86 ; 123.38)$ & 0.328 \\
\hline \multirow{2}{*}{$\begin{array}{l}\text { LV stroke } \\
\text { volume (ml) }\end{array}$} & Baseline & $36.26(32.09 ; 39.54)$ & $35.92(32.24 ; 38.42)$ & 0.878 \\
\hline & Explantation & $25.11(24.25 ; 30.59)$ & $32.51(29.30 ; 38.53)$ & 0.050 \\
\hline \multirow{2}{*}{$\begin{array}{l}\text { LV ejection } \\
\text { fraction }(\%)\end{array}$} & Baseline & $42.26(41.28 ; 49.29)$ & $46.89(44.55 ; 48.10)$ & 0.065 \\
\hline & Explantation & $29.78(25.00 ; 31.81)$ & $30.22(29.47 ; 35.35)$ & 0.574 \\
\hline \multicolumn{2}{|l|}{$\begin{array}{l}\text { Infarct volume } \\
\left(\mathrm{cm}^{3}\right)\end{array}$} & $9.28(5.45 ; 10.45)$ & $8.79(8.19 ; 11.02)$ & 1.000 \\
\hline \multicolumn{2}{|l|}{ LV volume $\left(\mathrm{cm}^{3}\right)$} & $78.24(75.86 ; 87.21)$ & $90.42(84.69 ; 98.04)$ & 0.065 \\
\hline \multicolumn{2}{|l|}{ LV mass (g) } & $81.36(78.89 ; 90.70)$ & $94.03(88.08 ; 101.96)$ & 0.065 \\
\hline \multicolumn{2}{|l|}{$\begin{array}{l}\text { LV mass/body weight } \\
(\mathrm{g} / \mathrm{kg})\end{array}$} & $2.16(2.04 ; 2.37)$ & $2.39(2.29 ; 2.47)$ & 0.093 \\
\hline \multicolumn{2}{|l|}{$\begin{array}{l}\text { Infarct volume, vs. LV } \\
\text { volume }(\%)\end{array}$} & $10.10(6.53 ; 10.91)$ & $9.20(7.71 ; 10.97)$ & 0.937 \\
\hline
\end{tabular}

Inf, infarction; reperf, reperfusion; LV, left ventricular.

in the serum of the control animals, the dronedarone and SR35021 concentrations were below the limit of detection.

Hemodynamic parameters. No statistically significant differences were identified in the heart rate or mean arterial blood pressure between the animals treated with or without dronedarone. In the two groups, the heart rate increased by $\sim 40 \%$ during reperfusion of the infarcted area, compared with baseline. The mean arterial blood pressure decreased in the two groups by $\sim 30 \%$ during MI and reperfusion, compared 

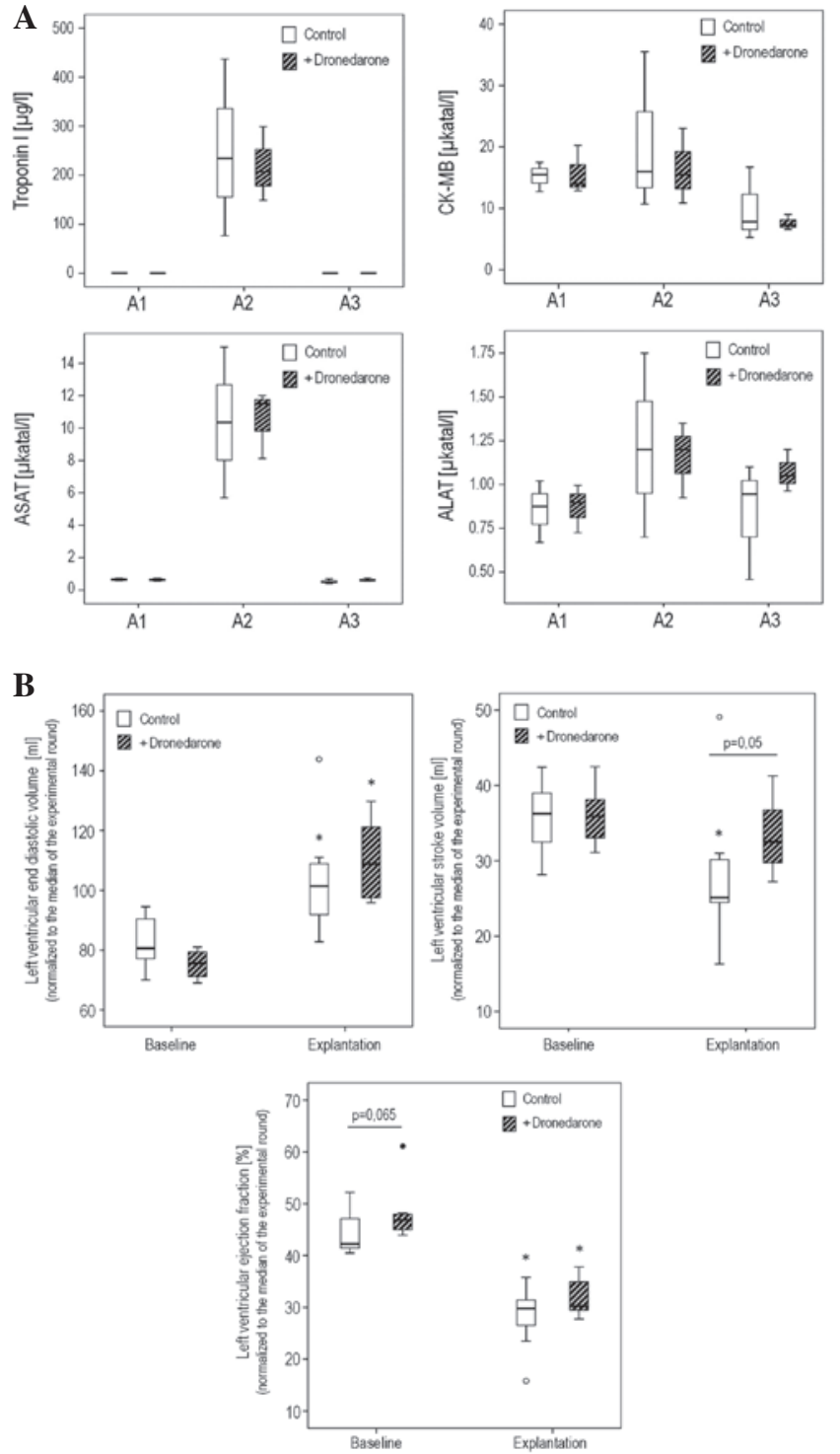

Figure 2. Clinical and functional parameters of myocardial infarction. (A) Plasma levels of Troponin I, CK-MB, ASAT, and ALAT prior to LAD occlusion (A1), 60 min after reperfusion (A2), and 28 days after infarction (A3). In response to infarction, there was an increase in the plasma levels of Troponin I, ASAT and CK-MB. No significant differences were identified in the plasma levels between the controls (A1, A2, and A3) and dronedarone-treated $(\mathrm{A} 1+\mathrm{D}, \mathrm{A} 2+\mathrm{D}, \mathrm{A} 3+\mathrm{D})$ pigs. (B) LVEDV, LVSV and LVEF on the day of infarction and explantation in the control and dronedarone-treated animals. LVEDV, LVSV, and LVEF were determined using left ventricular angiography ( $\mathrm{P}<0.05$, compared with baseline; values normalized to the median of the experimental round; $\mathrm{n}=8$ ). ALAT, alanine transaminase; ASAT, aspartate aminotransferase; LVEF, left ventricular ejection fraction; CK-MB, creatine kinase-MB; LVEDV, left ventricular end-diastolic volume; LVSV, left ventricular stroke volume.

with baseline. Dronedarone treatment did not impair the stability of the mean arterial blood pressure or increase the risk of ventricular arrhythmias and tachycardia during MI and reperfusion (Table I).

Compared with baseline, the dronedarone-treated animals and the control animals exhibited a significant prolongation of the QRS-duration on the resting ECG following 4 weeks of infarct healing (control, 55 vs. $60 \mathrm{~ms}$; dronedarone, 57 vs. $60 \mathrm{~ms}$, respectively; $\mathrm{P}=0.012$ ) and of the QTc-duration (adjusted to the heart rate; control,
362 vs. 399 ms; dronedarone, 376 vs. 410 ms, respectively; $\mathrm{P}=0.025)$. Dronedarone treatment had no significant effect on the PQ-, QRS- or QT-durations on the resting ECGs of the pigs.

Plasma markers of MI. As expected, the plasma levels of TnI, CK-MB, and ASAT were significantly increased in response to MI (blood sample \#A2; Fig. 1). All parameters decreased to baseline 28 days after infarction. No differences were identified in plasma levels between the dronedarone-treated group and the control group (Fig. 2A).

The data indicated that the severity of infarction/tissue damage induced by 90 min LAD occlusion did not differ between the groups.

$L V$ function. LV end-diastolic volume, stroke volume and ejection fraction were determined as parameters for LV function using LV angiography.

All animals in the control group exhibited a statistically significant decrease in LV stroke volume by an average of $31 \%$ following 4 weeks of infarct healing, as a result of impaired myocardial contraction in the area of the infarct $(\mathrm{P}=0.021)$. However, the stroke volume of the dronedarone-treated pigs did not decrease significantly on the day of the explantation, compared with the baseline. Therefore, LV stroke volume tended to increase by $7.4 \mathrm{ml}$ in the dronedarone-treated group, compared with the stroke volume of the control group, following 4 weeks of infarct healing $(\mathrm{P}=0.05)$.

Prior to experimental MI, the dronedarone-treated animals were observed to have a reduced end-diastolic volume, compared with the control animals, although this was not significant $(\mathrm{P}=0.105)$. As a result of $\mathrm{LV}$ dilatation, the end-diastolic volume of the dronedarone-treated animals was significantly increased by $44 \%$ following 4 weeks of infarct healing $(\mathrm{P}=0.012)$. The end-diastolic volume of the control animals was also significantly increased, compared with baseline, although only by $26 \%(\mathrm{P}=0.017)$. Following 4 weeks of infarct healing, the end-diastolic volumes of the control animals and the dronedarone-treated animals were similar in size at 101.4 and $108.88 \mathrm{ml}(\mathrm{P}=0.328$; Fig. 2B).

Compared with baseline measurements, the LVEF was significantly reduced by $30 \%$ in the control group $(\mathrm{P}=0.012)$ and $35 \%$ in the dronedarone-treated group $(\mathrm{P}=0.012)$ following 4 weeks of infarct healing (control, 42.26 vs. $29.78 \%$; dronedarone, 46.89 vs. $30.22 \%$ ). However, the dronedarone-treated animals tended to have a higher ejection fraction prior to experimental MI $(\mathrm{P}=0.065)$, although this was not detected following 4 weeks of infarct healing, However, dronedarone treatment did not impair LV function 4 weeks after experimental MI (Fig. 2B).

Histopathology. Evidence of an MI was present in all the hearts. The infarcted myocardium was replaced by granulation tissue and young fibrosis, corresponding to the alterations, which are expected 4 weeks after ischemia, termed 'myocardial infarct in organization' (17). The adjacent myocardium was vital and several cardiomyocytes were hypertrophic. Post-infarct edema was observed in all control and dronedarone-treated porcine hearts. 
A
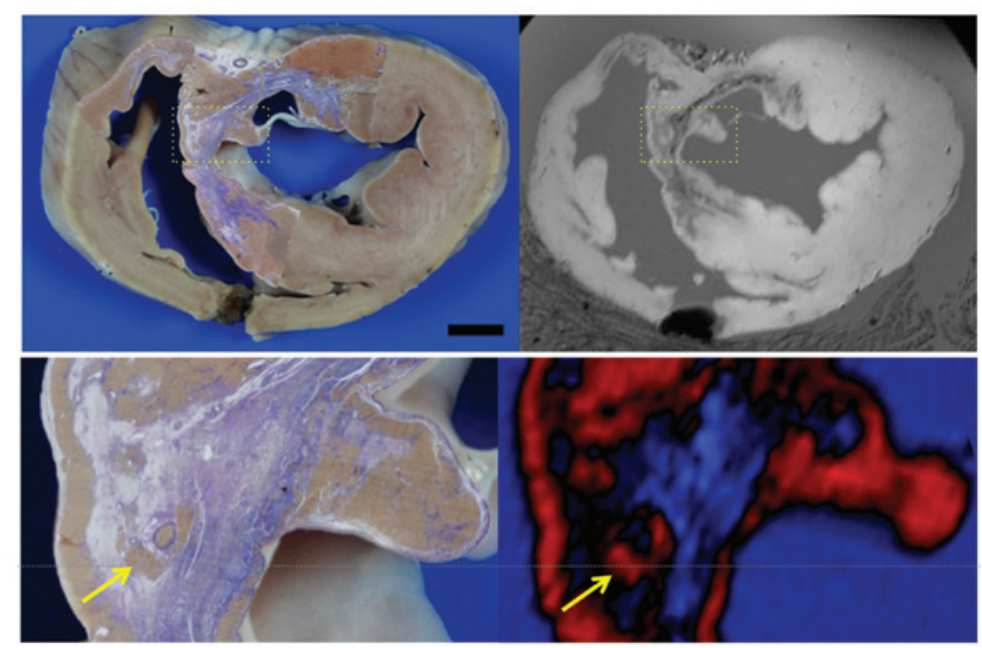

B

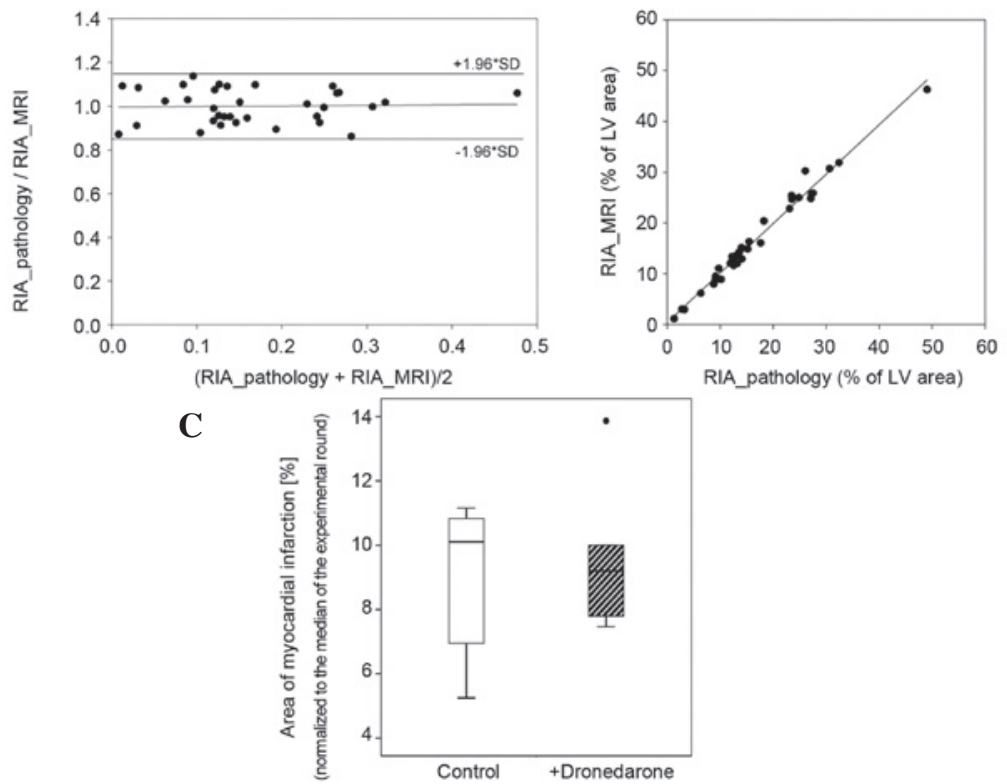

Figure 3. Infarct size determination. (A) Corresponding images of pathohistology and MRI. Images of acid fuchsin orange G-stained histological slices were pasted over the macroscopic image ( $3 \mathrm{~A}$, top; scale bar, $1 \mathrm{~cm}$ ). The corresponding plane of the MRI was aligned for comparison of the appearance of different tissue areas in MRI and histology. Heterogeneity of the myocardial infarct could be visualized by MRI as presented in the details, arrows marking islands of vital myocardial tissue in the infarcted area, as they were identified by histology (3A, bottom; 5.5x magnification of top row). (B) Bland-Altmann plot (left) and correlation (right) of the measurements of the RIA by MRI imaging versus histopathology, $(n=40)$. (C) Area of the myocardial infarct relative to the left ventricular volume. Volumes of the left ventricle and the left ventricular myocardial infarct were measured on the bases of the MRI images using Osirix software. The area of myocardial infarction was calculated as the quotient of the absolute infarct volume and the left ventricular volume, and is compared with and without dronedarone treatment. Values are normalized to the median of the experimental round $(\mathrm{n}=6)$. MRI, Magnetic resonance imaging; LV, left ventricular; RIA, relative infarct area.

Evaluating myocardial infarct size using 3D MRI. Infarcted regions were identified, even without the use of contrast agent, on MRI (Fig. 3A). In line with the corresponding histological specimens, it was demonstrated that MRI was able to detect viable myocardium in the infarct region (Fig. 3A). Vital regions inside the infarct zone were mostly found in the first layers of the subendocardial myocardium, but also in other areas of the infarcted area. The right ventricular myocardium in the region of the sulcus interventricularis was preserved. This was clearly delineated in the MRI and in the AFOG-stained histological sections. Thus, this region was used as a reference for vital myocardium inside the infarct zone. Measurement of the area of infarction in the pathological specimens and the corresponding MRI sections revealed a high level of correlation between the two methods
$\left(\mathrm{R}^{2}=0.98\right)$. In addition, no proportional bias was noted between the percentage of LV infarct areas, calculated from the segmented images obtained by MRI and histology, as demonstrated by the corresponding Bland-Altman analysis (Fig. 3B). The average inter-individual variation in the measured infarct size was $5.91 \%$.

The shape of the left ventricle on the MRI compared with the angiography demonstrated that the configuration of the hearts in vitro was similar to the shape of end-systolic LV.

The determination of the LV infarct size revealed no differences between the dronedarone group and the control group. Neither the absolute infarct volume nor the infarct size normalized to the LV volume were significantly altered by dronedarone treatment. Dronedarone did not enlarge the area affected by MI (Fig. 3C). 


\section{Discussion}

Coronary heart disease (CHD) is common among patients with $\mathrm{AF}(26)$ and contributes to an increased risk for cardiovascular events. In addition, CHD promotes ventricular arrhythmia, limiting the use of antiarrhythmic drugs in a particular fraction of AF patients with concomitant CHD. Dronedarone has been demonstrated to largely prevent the development of microcirculatory abnormalities in the ventricles, which is observed following a few hours of rapid atrial pacing (7). This is suggestive of dronedarone being particularly effective in the early stages of AF. Dronedarone reduces the recurrence of AF in patients with paroxysmal and persistent AF (27). The ATHENA trial revealed that dronedarone reduced cardiovascular hospitalization and mortality rates in patients with paroxysmal and persistent $\mathrm{AF}$ with $\geq 1$ risk factor for vascular events (2). Post-hoc analysis of a 2.5 year follow-up of the ATHENA trial analyzed the safety and cardiovascular outcome of dronedarone in $>1,400$ patients with persistent or paroxysmal AF and concomitant CHD. As observed previously in the ATHENA trial, dronedarone reduced cardiovascular hospitalization and mortality rates, and reduced first acute coronary syndrome (3). Of note, no increase of proarrhythmias in the dronedarone group was observed. In contrast to these positive effects of dronedarone, the PALLAS study had to be terminated early due to the risk of stroke, MI, systemic embolism or fatalities in patients with permanent $\mathrm{AF}$, who were treated with dronedarone. Therefore, dronedarone is not recommended for patients with permanent $\mathrm{AF}$ at high risk for cardiovascular events or in patients with severe heart failure (New York Heart Association class $\geq$ III) (28). The reasons for the discrepancy remain to be fully elucidated, however, it is reasonable to suggest that particularly advanced structural alterations of the myocardium in permanent AF and severe heart failure contribute to the lack of beneficial effects of dronedarone. A previous study reporting data from 4,856 Swedish patients with symptomatic, non-permanent AF treated with dronedarone revealed that the mortality rates in the patients treated with dronedarone were lower than in the AF patients who did not receive dronedarone. This effect remained following adjustment for a number of baseline variables (29). Hohnloser et al (30) also noted that the prescription of dronedarone to patients with AF does not expose patients to an increased risk of mortality or liver disease.

Experimental data has suggested that dronedarone may prevent microcirculatory flow abnormalities in the ventricles during AF (7). These flow abnormalities cause angina pectoris in patients following the initiation of an $\mathrm{AF}$ episode. Furthermore, microcirculatory alterations may lead to low grade ischemia, causing the release of troponin in the systemic circulation. The protective effects of dronedarone in the case of ischemia are not limited to AF-dependent changes of the microcirculatory system, rather they appear to reflect a more general, pleiotropic action of dronedarone $(14,15,24)$. Accordingly, dronedarone has been reported to exhibit protective activity in an acute MI model (24), in which dronedarone reduces the size of the infarcted tissue in an acute setting. It remained to be elucidated, however, whether the potential protective effects of dronedarone in reducing infarct size and preservng LV performance, are maintained long-term following an induced MI. The existence of longer-lasting effects is suggested by evidence that, in a rat model of cerebral infarction, a reduction in infarct size in the dronedarone-treated groups is observed 7 days post-infarction (31). This effect, however, was obtained with 3-7.5-fold higher dosages of dronedarone than those used in the present study. Notably, these beneficial effects of dronedarone on infarct size remained, although to a lesser extent, when dronedarone was administered $2 \mathrm{~h}$ following infarction. This indicates that dronedarone affects different phases and mechanisms during infarction in repair. Previous studies have indicated that the protective mechanisms may include the improvement/preservation of microvascular flow (7), reduction of heart rate (32) and direct cardioprotection, which may result from the induction of a redox-associated gene expression panel (7) or the protection from calcium-overload $(16,19)$. It has been suggested that the first effect may be partly due to the attenuation of AF-dependent $\alpha$-adrenergic vasoconstriction $(16,33)$. In an acute infarction model, the reduction in infarct size by dronedarone $2 \mathrm{~h}$ following reperfusion was found to be independent of the remaining blood flow (16).

In an experimental rat model, dronedarone has been revealed to reduce the incidence of ischemia- and reperfusion-induced arrhythmia and mortality rates (34). This may be due to a reduction of extrasystoles, as was observed in a rabbit right ventricle 'border zone' model (35). A meta-analysis of the effects of dronedarone on the occurrence of stroke or transient ischemic attack indicated a reduced risk in patients with paroxysmal and persistent $\mathrm{AF}$ treated with dronedarone (36). Of note, a previous study also demonstrated the potential of a combined administration of low dose dronedarone and ranolazine together reducing ischemia-induced AF vulnerability and AF duration, as well as ventricular tachyarrhythmias (37).

In conclusion, data from experimental and clinical studies indicate the existence of acute beneficial effects of dronedarone in the setting of ischemia/reperfusion, although the underlying mechanisms remain to be fully elucidated. The results of the present study do not support the hypothesis that dronedarone exerts beneficial long-term effects ( 28 days post infarct) on infarct size following acute MI. In the present study, the area of the MI was not associated with the area at risk, as it was intended to preserve the tissue for further gene expression investigations, which may have contributed to the discrepancy between the observations. LAD occlusion, however, was performed by highly experienced personnel following a standardized protocol to minimize experimental variation. The LAD was occluded directly following the first diagonal branch. More proximal occlusion of the LAD directly following the left main coronary artery is known to produce refractory ventricular fibrillation in pigs, and, therefore, the aforementioned technique and location was selected. Of note, other investigations using the same experimental protocol of LAD occlusion did not detect a significant variation in the size of the area at risk (16). Another limitation of the present study is the relatively small number of animals included, which is the reason the data required careful interpretation. However, the data suggested, that the use of dronedarone is safe for patients with $\mathrm{AF}$ who suffer an acute anterior MI. 
How dronedarone affects myocardial gene expression in healthy, infarcted or border zone myocardium and how it may improve myocardial function and stability in the long-term, remain to be elucidated in future investigations.

In the present study, in vitro MRI was used for the determination of infarct size, in addition to in vivo angiography. In vitro MRI for the quantification of pathological changes is an expanding field, as high-resolution 3D data sets may be acquired and analyzed at an almost microscopic level. Usually, contrast media are applied to delineate the pathological tissue, however, as they are taken up by the cells, changes in gene expression may occur, including monocytes (38), and distort results of interest.

In the present study, it was demonstrated that, even without the use of contrast agents, in vitro MRI is a suitable approach to delineate infarcted regions from vital myocardium, as judged in comparison to the pathohistology. In several sections, the first layers of the subendocardial myocardium were found to be vital. This was clearly delineated by the MRI and in the AFOG-stained histological sections. Not only this subendocardial vital zone, but also intermingled areas of viable cells surrounded by fibrosis were visualized in the MRI. Thus, compared with the pathohistological investigations, the complete pig heart can be readily analyzed in sections as small as $1 \mathrm{~mm}$, or even less, producing accurate data on infarct size. In the present study, no reduced scar formation was detected in the dronedarone-treated animals, which is in accordance with the angiographic finding that EF was reduced to almost the same degree in the control and dronedarone group.

In summary, the results of the current study demonstrate the suitability of MRI for infarct size determination without the need for contrast agents. Although we a reduction of infarct size by dronedarone was not observed, the data suggest that dronedarone is not harmful in patients who suffer from acute anterior MI. Future studies are required to assess the consequences of dronedarone-dependent alterations in myocardial gene expression on cardiac structure and function in the long-term.

\section{Acknowledgements}

The authors would like to thank Mrs. Manja Möller and Mrs. Ines Schultz (Institute of Medical Biochemistry and Molecular Biology, University Medicine Greifswald, Ernst-Moritz-Arndt-University, Greifswald, Germany), Mrs.Cathleen Brandenburg and Mrs.Christine Karrer-Hoffmann (Institute of Pathology, University Medicine Greifswald, Ernst-Moritz-Arndt-University, Greifswald, Germany) and Mr. Stefan Hadlich (Department of Diagnostic Radiology and Neuroradiology, University Medicine Greifswald, Ernst-Moritz-Arndt-University, Greifswald, Germany) for their technical assistance. The authors would also like to thank Sanofi-Aventis for financial support of this investigator-sponsored trial.

\section{References}

1. Miyasaka Y, Barnes ME, Gersh BJ, Cha SS, Bailey KR, Seward JB, Iwasaka T and Tsang TS: Coronary ischemic events after first atrial fibrillation: Risk and survival. Am J Med 120: 357-363, 2007.

2. Hohnloser SH, Crijns HJ, van Eickels M, Gaudin C, Page RL, Torp-Pedersen C, Connolly SJ and ATHENA Investigators: Effect of dronedarone on cardiovascular events in atrial fibrillation. N Engl J Med 360: 668-678, 2009
3. Pisters R, Hohnloser SH, Connolly SJ, Torp-Pedersen C, Naditch-Brûlé L, Page RL, Crijns HJ and ATHENA Investigators; Effect of dronedarone on clinical end points in patients with atrial fibrillation and coronary heart disease: Insights from the ATHENA trial. Europace 16: 174-181, 2014.

4. Saito D, Haraoka S, Ueda M, Fujimoto T, Yoshida H and Ogino Y: Effect of atrial fibrillation on coronary circulation and blood flow distribution across the left ventricular wall in anesthetized open-chest dogs. Jpn Circ J 42: 417-423, 1978.

5. Wichmann J, Ertl G, Rudolph G and Kochsiek K: Effect of experimentally induced atrial fibrillation on coronary circulation in dogs. Basic Res Cardiol 78: 473-491, 1983.

6. Goette A, Bukowska A, Dobrev D, Pfeiffenberger J, Morawietz H, Strugala D, Wiswedel I, Röhl FW, Wolke C, Bergmann S, et al: Acute atrial tachyarrhythmia induces angiotensin II type 1 receptor-mediated oxidative stress and microvascular flow abnormalities in the ventricles. Eur Heart J 30: 1411-1420, 2009.

7. Bukowska A, Hammwöhner M, Sixdorf A, Schild L, Wiswedel I, Röhl FW, Wolke C, Lendeckel U, Aderkast C, Bochmann S, et al: Dronedarone prevents microcirculatory abnormalities in the left ventricle during atrial tachypacing in pigs. Br J Pharmacol 166: 964-980, 2012.

8. Yang KC and Dudley SC Jr: Oxidative stress and atrial fibrillation: finding a missing piece to the puzzle. Circulation 128: 1724-1726,2013.

9. Mayyas F, Alzoubi KH and Van Wagoner DR: Impact of aldosterone antagonists on the substrate for atrial fibrillation: Aldosterone promotes oxidative stress and atrial structural/electrical remodeling. Int J Cardiol 168: 5135-5142, 2013.

10. Bonilla IM, Sridhar A, Györke S, Cardounel AJ and Carnes CA: Nitric oxide synthases and atrial fibrillation. Front Physiol 3: 105, 2012.

11. Raddino R, Poli E, Pelà G, Gargano M and Manca C: Inhibitory actions of amiodarone on the isolated rabbit heart and aorta. Gen Pharmacol 20: 313-317, 1989.

12. Goette A, Bukowska A, Lillig CH and Lendeckel U: Oxidative stress and microcirculatory flow abnormalities in the ventricles during atrial fibrillation. Front Physiol 3: 236, 2012.

13. Hodeige D, Heyndrickx JP, Chatelain P and Manning A: SR 33589, a new amiodarone-like antiarrhythmic agent: Anti-adrenoceptor activity in anaesthetized and conscious dogs. Eur J Pharmacol 279: 25-32, 1995.

14. Sobrado LF, Varone BB, Machado AD, Nearing BD, Zeng D, Belardinelli L and Verrier RL: Dronedarone's inhibition of If current is the primary mechanism responsible for its bradycardic effect. J Cardiovasc Electrophysiol 24: 914-918, 2013.

15. Heusch G: Heart rate in the pathophysiology of coronary blood flow and myocardial ischaemia: Benefit from selective bradycardic agents. Br J Pharmacol 153: 1589-1601, 2008.

16. Skyschally A and Heusch G: Reduction of myocardial infarct size by dronedarone in pigs-a pleiotropic action? Cardiovasc Drugs Ther 25: 197-201, 2011.

17. Blankesteijn WM, Creemers E, Lutgens E, Cleutjens JP, Daemen MJ and Smits JF: Dynamics of cardiac wound healing following myocardial infarction: Observations in genetically altered mice. Acta Physiol Scand 173: 75-82, 2001.

18. Plank G, Burton RA, Hales P, Bishop M, Mansoori T, Bernabeu MO, Garny A, Prassl AJ, Bollensdorff C, Mason F, et al: Generation of histo-anatomically representative models of the individual heart: tools and application. Philos Trans A Math Phys Eng Sci 367: 2257-2292, 2009.

19. Pop M, Ghugre NR, Ramanan V, Morikawa L, Stanisz G, Dick AJ and Wright GA: Quantification of fibrosis in infarcted swine hearts by ex vivo late gadolinium-enhancement and diffusion-weighted MRI methods. Phys Med Biol 58: 5009-5028, 2013.

20. Fridericia LS: Die Systolendauer im Elektrokardiogramm bei normalen Menschen und bei Herzkranken. Acta Med Scand 53: 469-486, 1920 (In German).

21. Bergmeyer HU, Scheibe P and Wahlefeld AW: Optimization of methods for aspartate aminotransferase and alanine aminotransferase. Clin Chem 24: 58-73, 1978.

22. Neumeier D, Prellwitz W, Würzburg U, Brundobler M, Olbermann M, Just H-J, Knedel M and Lang H: Determination of creatine kinase isoenzyme MB activity in serum using immunological inhibition of creatine kinase M subunit activity. Activity kinetics and diagnostic significance in myocardial infarction. Clin Chim Acta 73: 445-451, 1976. 
23. Ullman EF, Kirakossian H, Singh S, Wu ZP, Irvin BR, Pease JS Switchenko AC, Irvine JD, Dafforn A, Skold CN, et al: Luminescent oxygen channeling immunoassay: Measurement of particle binding kinetics by chemiluminescence. Proc Natl Acad Sci USA 91: 5426-5430, 1994.

24. Goubran M, Crukley C, de Ribaupierre S, Peters TM and Khan AR: Image registration of ex-vivo MRI to sparsely sectioned histology of hippocampal and neocorticatemporal lobe specimens. Neuroimage 83: 770-781, 2013.

25. Shah DJ, Kim HW, James O, Parker M, Wu E, Bonow RO, Judd RM and Kim RJ: Prevalence of regional myocardial thinning and relationship with myocardial scarring in patients with coronary artery disease. JAMA 309: 909-918, 2013.

26. Nieuwlaat R, Capucci A, Camm AJ, Olsson SB, Andresen D, Davies DW, Cobbe S, Breithardt G, Le Heuzey JY, Prins $\mathrm{MH}$, et al: Atrial fibrillation management: a prospective survey in ESC member countries: The Euro Heart Survey on Atrial Fibrillation. Eur Heart J 26: 2422-2434, 2005.

27. Singh BN, Connolly SJ, Crijns HJ, Roy D, Kowey PR Capucci A, Radzik D, Aliot EM, Hohnloser SH; EURIDIS and ADONIS Investigators: Dronedarone for maintenance of sinus rhythm in atrial fibrillation or flutter. N Engl J Med 357: 987-999, 2007.

28. Camm AJ, Lip GY, De Caterina, R, Savelieva I, Atar D, Hohnloser SH, Hindricks G and Kirchhof P: ESC Committee for Practice Guidelines-CPG; Document Reviewers: 2012 focused update of the ESC Guidelines for the management of atrial fibrillation: an update of the 2010 ESC Guidelines for the management of atrial fibrillation-developed with the special contribution of the European Heart Rhythm Association. Europace 14: 1385-1413, 2012 .

29. Friberg L: Safety of dronedarone in routine clinical care. J Am Coll Cardiol 63: 2376-2384, 2014.

30. Hohnloser S: Dronedarone: 'Real-world' data vis-à-vis data from randomized clinical trials. J Am Coll Cardiol 63: 2385-2387, 2014.
31. Engelhorn T, Schwarz MA, Heusch G, Doerfler A and Schulz R: Reduction of cerebral infarct size by dronedarone. Cardiovasc Drugs Ther 25: 523-529, 2011.

32. Finance O, Manning A and Chatelain P: Effects of a new amiodarone-like agent, SR 33589, in comparison to amiodarone, $\mathrm{D}, \mathrm{L}$-sotalol and lignocaine, on ischemia-induced ventricular arrhythmias in anesthetized pigs. J Cardiovasc Pharmacol 26: 570-576, 1995.

33. Hodeige D, Heyndrickx JP, Chatelain P and Manning A: SR 33589, a new amiodarone-like antiarrhythmic agent: Anti-adrenoceptor activity in anaesthetized and conscious dogs. Eur J Pharmacol 279: 25-32, 1995.

34. Manning AS, Bruyninckx C, Ramboux J and Chatelain P: SR 33589, a new amiodarone-like agent: Effect on ischemiaand reperfusion-induced arrhythmias in anesthetized rats. J Cardiovasc Pharmacol 26: 453-461, 1995.

35. Alexandre J, Rouet R, Puddu PE, Beygui F, Manrique A and Milliez P: Dronedarone versus amiodarone in preventing premature ventricular contractions in an in vitro model of 'border zone.' J Cardiovasc Pharmacol 63: 49-57, 2014.

36. Dagres N, Varounis C, Iliodromitis EK, Lekakis JP, Rallidis LS and Anastasiou-Nana M: Dronedarone and the incidence of stroke in patients with paroxysmal or persistent atrial fibrillation: A systematic review and meta-analysis of randomized trials. Am J Cardiovasc Drugs 11: 395-400, 2011.

37. Verrier RL, Pagotto VP, Kanas AF, Sobrado MF, Nearing BD, Zeng D and Belardinelli L: Low doses of ranolazine and dronedarone in combination exert potent protection against atrial fibrillation and vulnerability to ventricular arrhythmias during acute myocardial ischemia. Heart Rhythm 10: 121-127, 2013.

38. Del Galdo F, Wermuth PJ, Addya S, Fortina P and Jimenez SA: $\mathrm{NF} \kappa \mathrm{B}$ activation and stimulation of chemokine production in normal human macrophages by the gadolinium-based magnetic resonance contrast agent Omniscan: Possible role in the pathogenesis of nephrogenic systemic fibrosis. Ann Rheum Dis 69: 2024-2033, 2010 